\section{Objects and Classes}

CS211

Fall 2000

\section{Much of Java Looks Like C}

- Arithmetic \& relationa operators are the same:

- Goal was to make a programming language that people would pick up easily - Assignment is the same: $\mathrm{a}=\mathrm{b}$;

- There are lots of $C$ and $\mathrm{C}++$ programmers, so make it much like $\mathrm{C}$

- Conditional \& looping statements are the same: if/else, while, for, do, break, continue, switch

- Arrays are the same: $a[i]$ and $b[i][i]$

\section{What's Different?}

- Java allows method overloading

- $\mathrm{C}++$ does this, but $\mathrm{C}$ does not

- $\mathrm{C}++$ also allows operator overloading; Java does not

- The Java numeric types all conform to IEEE standards

- C numeric types can vary depending on platform

- Java does not have explicit pointers

- In Java, there is a separate String class

- A String is not the same as an array of characters and it is not terminated by the NUL character

- Java does automatic Garbage Collection

- Many other differences...

- Java is claimed to be safer more portable, and easier to use than $\mathrm{C}++$

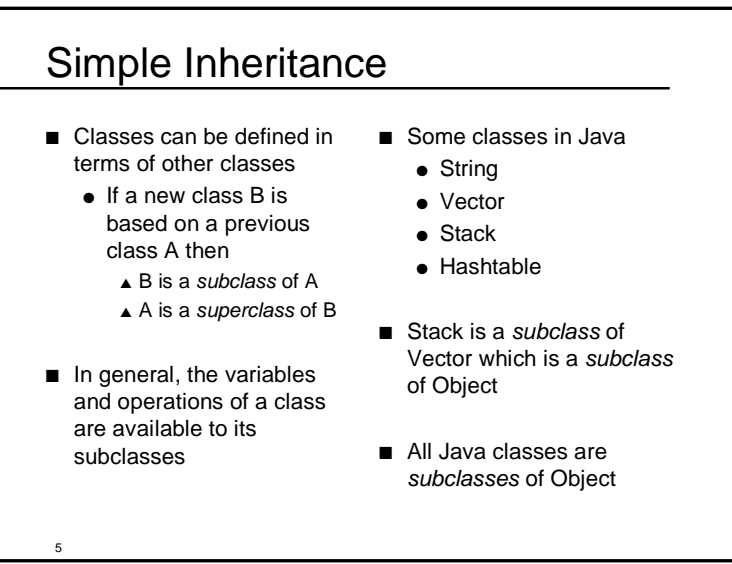

\section{Object Oriented Programming}

- This is a style of programming based on the ideas of

- Objects

- Classes

- Inheritance

- Java is based on these ideas

a Currently, this is the best of known programming styles

- An object is a software bundle of data and related operations (the operations are called methods in Java)

- A class is a template that defines objects of a certain kind

- Using one class, I can create several objects where each is an instance of this class

\title{
Java Programs
}

- A Java program consists of a number of interacting classes

- All methods and all variables reside within some class

- When an application runs

- You specify a class

- The "system" looks for and runs the method that looks like

A public static void main(String[ ] args) 


\section{Java Programs: Applets}

- When a Java Applet runs

- The web page specifies a class

- The "system" looks for these methods $\Delta$ public void init()

v Runs when Applet is first loaded $\Delta$ public void start()

v Runs when Applet appears on screen

$\Delta$ public void stop()

v Runs when Applet is off screen

$\Delta$ public void destroy()

Runs when Applet is terminating

\section{Object Basics}

- Primitive types in Java:

- byte, short, int, long

- float, double

- char

- boolean

- Everything else is an Object

- Each object is an instance of a Java class

- There are many

predefined Java classes

- Operators (with one exception) work only on primitive types

- What's the exception?

- Each Java variable holds one of two things:

- a primitive type or

- a reference to an object

\section{Some Terminology}

\begin{tabular}{|c|c|c|}
\hline \multirow{4}{*}{$\begin{array}{l}\text { public class Thing \{ } \\
\text { private int value; } \\
\text { public static int count; } \\
\text { public void setValue (int v) \{ value }=\mathrm{v} ;\} \\
\text { public int getValue }(\text { ) \{ return value; }\} \\
\text { // Plus other methods } \\
\text { \} }\end{array}$} & \multicolumn{2}{|c|}{$\begin{array}{l}\text { Thing } \mathrm{t}=\text { new Thing }(\text { ); } \\
\text { is executed, an object is } \\
\text { created that looks like this }\end{array}$} \\
\hline & Thing & $\_$class name \\
\hline & $\begin{array}{l}\text { value } \\
\text { count }\end{array}$ & $\longleftarrow$ attributes \\
\hline & $\begin{array}{l}\text { getValue() } \\
\text { setValue() }\end{array}$ & $\longleftarrow$ methods \\
\hline
\end{tabular}

Warning: The picture suggests that each object gets its own copy of each method. This provides some good intuition, but is not really true...

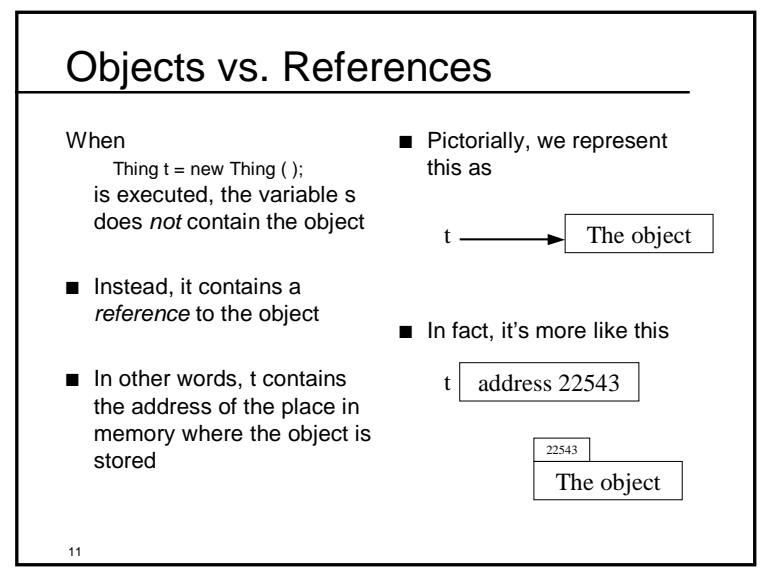

\section{Object vs. Reference Example}

What happens?

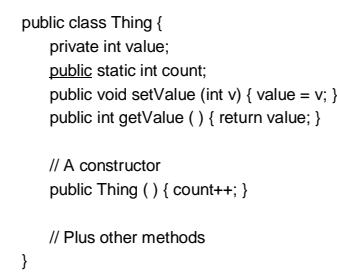

\}

- function vs. procedure?

- accessor methods vs. modifier methods? 


\section{Null}

- What happens after the declaration, but before the assignment?

Thing t1;

// What has happened here?

$\mathrm{t} 1$ = new Thing $($ );

- The variable $t 1$ exists, but it contains no reference

- It holds the special value null

- null can be assigned to any object variable

- null can be used in "==" tests

\section{Equality}

- The "==" operator in Java tests whether two variables contain the same value

- For primitive types, this is what we want

- For objects, this

compares "addresses"

What happens?

Thing $\mathrm{t} 1=$ new Thing $($ );

Thing $\mathrm{t} 2=$ new Thing (

t1. setValue(44);

t2.setValue(44);

System.out.println( $\mathrm{t} 1==\mathrm{t} 2$ ):

- Need an "equals( )" method that compares the contents of the object

\section{An Improved Thing class}

public class Thing \{

private int value;

public static int count;

public void setValue (int v) $\{$ value $=v$; $\}$

public int getValue () \{return value; \}

// A constructor

public Thing ( ) \{ count++;

// Equality test

public boolean equals (Thing other) \{

return value $==$ other.value:

// Plus other methods
- Every class automatically has an equals( ) method

- The default equals( )

method is inherited from Object

- This is usually not what you actually want

- You often need to write your own equals( )

\section{Assignment vs. Copying (Cloning)}

- What happens if we really want to make a copy of an object?

- Can't do it this way: Thing $\mathrm{t} 1 \mathrm{=}$ new $\operatorname{Thing}()$; // Do stuff with $\mathrm{t} 1$; now make a copy Thing $\mathrm{t} 2$ = new Thing $($ ); $\mathrm{t} 2=\mathrm{t} 1$

- Instead we use the "clone( )" method: Thing $\mathrm{t} 2=\mathrm{t}$. clone () ;

- Can use inherited (from Object) clone ( ) if class

Thing implements Cloneable public class Thing \{

private int value;

public static int count:

public void setValue (int $v$ ) $\{$ value $=v$;

public int getValue () \{ return value; public Thing () \{ count++; $\}$ public boolean equals (Thing other) \} return value $==$ other value ;

public Thing clone ( ) \{ Thing thing = new Thing ( ) thing.value $=$ getValue $($ ) return thing; 